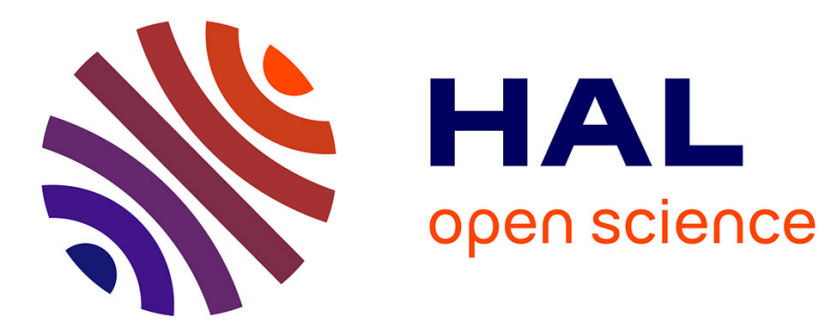

\title{
Robust expansion of experimental mode shapes under epistemic uncertainties
}

\author{
Antoine Kuczkowiak, Scott Cogan, Morvan Ouisse, Emmanuel Foltête, \\ Mathieu Corus
}

\section{- To cite this version:}

Antoine Kuczkowiak, Scott Cogan, Morvan Ouisse, Emmanuel Foltête, Mathieu Corus. Robust expansion of experimental mode shapes under epistemic uncertainties. International Modal Analysis Conference, Feb 2014, Orlando, FL, United States. pp.419-427, 10.1007/978-3-319-04552-8_42 . hal02300559

\section{HAL Id: hal-02300559 \\ https://hal.science/hal-02300559}

Submitted on 29 Sep 2019

HAL is a multi-disciplinary open access archive for the deposit and dissemination of scientific research documents, whether they are published or not. The documents may come from teaching and research institutions in France or abroad, or from public or private research centers.
L'archive ouverte pluridisciplinaire HAL, est destinée au dépôt et à la diffusion de documents scientifiques de niveau recherche, publiés ou non, émanant des établissements d'enseignement et de recherche français ou étrangers, des laboratoires publics ou privés.

\section{(c)(1)}

Distributed under a Creative Commons Attribution| 4.0 International License 


\title{
Robust expansion of experimental mode shapes under epistemic uncertainties
}

\author{
A. Kuczkowiak ${ }^{*(1,2)}$, S. Cogan ${ }^{(1)}$, M. Ouisse ${ }^{(1)}$, E. Foltête $^{(1)}$, and M. Corus ${ }^{(2)}$ \\ ${ }^{(1)}$ Department of Applied Mechanics, FEMTO-ST Institute - 24, rue de l'Épitaphe, 25000 Besançon, France \\ ${ }^{(2)}$ Department of Mechanic and Acoustic Analysis, EDF R\&D - 1, avenue du Général de Gaulle, 92141 Clamart, France
}

\begin{abstract}
Important variations in response behaviors of power plant generators are observed in a population of nominally identical installations due to numerous and significant sources of variability. As a result, it proves to be extremely difficult to implement a predictive and reliable physics-based model. The present study attempts to leverage an existing non validated numerical model to reconstruct information on unobserved degrees of freedom based on the results of modal tests. An expansion method is proposed based on the concept of the constitutive relation error (CRE). This method leads to minimization of an energy-based functional that takes into account both errors in the model and in the test data. Due to lack of knowledge, commonplace in this kind of complex system, the expansion will be presented in the framework of robust approach. More precisely, the first objective of this article is to assess the robustness of the mode shape expansion in presence of large epistemic uncertainties that are represented as info-gap models. Secondly, a strategy will be presented to maximize the robustness of the expansion by appropriately selecting the model decision variables for a given horizon of uncertainty. The proposed methodology is illustrated on simple academic test cases.
\end{abstract}

\section{Keywords}

Expansion methods, robust calibration, uncertainty, info-gap theory, constitutive relation error.

\section{Introduction}

Expansion methods are used to extend incomplete measured data set on a discretized numerical model. More precisely, mode shape expansion techniques seek to obtain the unmeasured part of the experimentally identified eigensolutions known only on a limited number of sensors. Mode shape expansion has many applications such as for test-analysis correlation and model calibration studies, for model error localization [13], for use in a hybrid model [5], for damage assessment [17], etc. In this article, an expansion method is used with the objective to construct a hybrid model. Numerous and significant sources of variability observed in a population of nominally identical power plant alternator, the structure of interest in this work, prevent to implement a predictive and reliable physics-based model. A finite-element (FE) model of the generator is available but non-validated since the numerical eigensolutions mismatch with the experimental one. Furthermore, a numerical model, projected onto the FE discretization - composed of $N$ degrees of freedom (dof)-, of the electromagnetic load in the air gap, between the rotor and the stator and denoted $\{F\}$, permits to compute the response levels $\left\{q^{n}\right\}$ in operation at the frequency of interest. Indeed, the response levels can be assessed by the hybrid model:

$$
\left\{q^{n}\right\}=[\hat{\Phi}]\left(-\omega^{2}\left[\mathcal{I}_{d}\right]+2 j \omega\left[\Lambda^{t}\right]\left[D^{t}\right]+\left[\Lambda^{t}\right]^{2}\right)^{-1}[\hat{\Phi}]^{T}\{F\} \in \mathbb{C}^{N},
$$

where $\left[\Lambda^{t}\right] \in \mathbb{R}^{m, m}$ is the identified spectral matrix composed of the $m$ identified eigenfrequencies, $\left[D^{t}\right] \in \mathbb{R}^{m, m}$ is the diagonal modal damping matrix obtained experimentally, $[\hat{\Phi}] \in \mathbb{R}^{N, m}$ is the expanded modal matrix and $\left[\mathcal{I}_{d}\right] \in \mathbb{R}^{m, m}$ is the identity matrix.

\footnotetext{
${ }^{*}$ Corresponding author antoine.kuczkowiak@femto-st.fr
} 
Since the eighties, various expansion methods are available (see $[2,14]$ for the state of the $\operatorname{art}^{1}$ ). Broadly speaking, mode shape expansion methods can be divided into two parts:

- Model-based expansion where the stiffness $[K]$ and mass $[M]$ matrices of the FE model are used to construct the unmeasured part of mode shapes, eg, the dynamic expansion [11]. The static expansion [9], based on Guyan reduction, expands the mode shapes with the main hypothesis that the inertial forces on the unmeasured dofs are ignored and thus this method is limited to low-frequency problems. The IRS method [15] allows the static expansion to be enhanced. An another class of methods use derived-model data: modal-based expansion techniques exploit the numerical modal basis as a subspace for expanding mode shape eg, SEREP /modal [16];

- Energy-based expansion where three different types of residues, weighted with an energy-norm, are minimized: output/input residues and residue on the constitutive relation. There are various expansion methods based on the minimization of error in the constitutive relation, for instance, the MDRE [1], the MECE [17, 18] or the CRE/ECRE $[12,20]$.

The first two methodologies share the same fundamental hypothesis: the numerical model must satisfy the stiffness and inertial properties of the real structure otherwise it will be tricky to obtain the real mode shapes. Thus, these methods depend strongly on the quality of the FE model. Energy-based expansion takes into account both error in the model and in the test data. That is why we use in this work energy-based expansion methods, namely based on the Extended Constitutive Relation Error (ECRE).

Minimization of errors committed during the expansion process depends always on the quality of the model, ie the ability of the model to represent test data. When dealing with FE model of industrial relevance, calibration strategies must be implemented before expanding mode shapes. The classical way is to reduce the discrepancy between the numerical model and the test data by appropriately selecting design variables in the numerical model. Various calibration strategies exist (non-parametric, local deterministic, local stochastic methods) but they all only focus to minimize the fidelity to data. Furthermore, in most of real world applications, the FE model is often polluted by various sources of lack of knowledge due to poorly understood physics. Local robust methods, presented in [4], aims to analyze the impact of lack of knowledge in the calibration process by defining three fundamental components:

- Fidelity to data: quantify the test-analysis discrepancy based on specify metric;

- Robustness to lack of knowledge: quantify the impact of lack of knowledge on the model prediction error;

- Prediction-looseness: quantify the confidence that can be accorded to the calibrated model given the range of model responses which are consistent with the lack of knowledge.

The inherent antagonistic nature of these components leads to fundamental trade-offs that forms the basis of the new model calibration paradigm. The reader can refer to $[10,19]$ for various illustrations of robust calibration strategy.

The present work aims to assess the robustness of the mode shape expansion in presence of large epistemic uncertainties that are represented as info-gap models. The second objective is to present a strategy, based on the concept of local robust method presented in [4], with the objective to maximize the robustness of the expansion. The section 2 introduces the proposed strategy based both on ECRE-based expansion and robust calibration and a numerical application is proposed in section 3 .

\section{Robust ECRE-based expansion}

\subsection{ECRE-based expansion: formulation}

In this article, a brief description of CRE concept is proposed. The reader can refer to [6, 7, 8] for a more in-depth description of CRE concepts. For the sake of clarity, we will consider that the model is erroneous only in term of stiffness. Let $(\{u\},\{v\})$, two displacement fields such as:

- $\{u\}$ is kinematically admissible, ie verifies the equilibrium equations;

\footnotetext{
${ }^{1}$ Detailed descriptions of these methods are out-of-scope of the paper.
} 
- $\{v\}$ is derived from the constitutive relation between the stress and the displacement such as the field $\{u-v\}$ expresses the error in stiffness in the model;

The FE discretization yields to the following energy-based functional:

$$
\xi_{\omega}^{2}(\{u\},\{v\})=\{u-v\}^{T}[K]\{u-v\}
$$

An extended formulation is proposed, namely ECRE [20], and includes test data $\{\tilde{u}\}$ :

$$
e_{\omega}^{2}(\{u\},\{v\},\{w\})=\xi_{\omega}^{2}(\{u\},\{v\},\{w\})+\frac{r}{1-r}\{\Pi u-\tilde{u}\}^{T}\left[K_{R}\right]\{\Pi u-\tilde{u}\},
$$

with $[\Pi]$, a projection operator from the FE model space to the observation space, $\left[K_{R}\right]$, the stiffness matrix of the nominal model reduced to the measurement $\operatorname{dofs}^{2}$ and $r \in[0 ; 1]$ a weighting coefficient allowing the relative confidence in the identified eigenvectors to be taken into account in the cost function $e_{\omega}^{2}$. The idea behind CRE concepts is to minimize the non-reliable data while relaxing admissibility constraints:

$$
\begin{cases}\text { minimize } & e_{\omega}^{2} \\ \text { under the constraint } & {[K]\{u-v\}-\left([K]-\omega^{2}[M]\right)\{u\}=0 .}\end{cases}
$$

To solve the problem (4), the constraint equations will be introduced via a vector of Lagrange multipliers $\{\lambda\}$. The optimization problem thus becomes:

$$
\min \left\{f(\{u\},\{v\},\{\lambda\})=e_{\omega}^{2}+\{\lambda\}^{T}\left([K]\{u-v\}-\left([K]-\omega^{2}[M]\right)\{u\}\right)\right\}
$$

Equation (5) is a saddle-point problem: the solution is defined by the stationarity conditions of the function $f$ with respect to the unknown vector $\{u\},\{v\}$ and $\{\lambda\}$ :

$$
\frac{\partial f}{\partial\{u\}}=0 \quad \frac{\partial f}{\partial\{v\}}=0 \quad \frac{\partial f}{\partial\{\lambda\}}=0
$$

Rearranging equations and using the fact that for any hermitian matrix $[M]$ and for any $\{x\} \in \mathbb{C}^{N}, \frac{\partial\{x\}[M]\{x\}}{\partial\{x\}}=$ $2[M]\{x\}$, equations (6) yields to the following linear system:

$$
[A]\{x\}=\{b\}
$$

where:

$$
\mid A]=\left[\begin{array}{cc}
-[K] & {[K]-\omega^{2}[M]} \\
{[K]-\omega^{2}[M]} & \frac{r}{1-r}[\Pi]^{T}\left[K_{R}\right][\Pi]
\end{array}\right] \quad\{x\}=\left\{\begin{array}{c}
u-v \\
u
\end{array}\right\} \quad \text { and } \quad\{b\}=\left\{\begin{array}{c}
\{0\} \\
\frac{r}{1-r}[\Pi]^{T}\left[K_{R}\right]\{\tilde{u}\}
\end{array}\right\} .
$$

The ECRE-based expansion involves to solve a linear system with the objective to find the unmeasured part of the identified eigensolutions, denoted $\{u\}$. The resolution of such system is performed directly in two main steps by using MD Nastran ${ }^{\circledR}$ language (DMAP language): decomposition coupled with a forward-backward substitution. However, solving such linear system yields to prohibitive CPU-time because of the decomposition process of $[A]$. The decomposition algorithms available in MD Nastran ${ }^{\circledR}$ are suitable for large, sparse, hermitian matrices with a small bandwidth of non-zero terms around the diagonal. However, ECRE equations lead to matrices with large bandwidth of non-zero terms around the diagonal (cf. Figure 1), which precisely makes the decomposition operation prohibitive.

\footnotetext{
${ }^{2}$ In practice, the Guyan stiffness matrix is often used.
} 

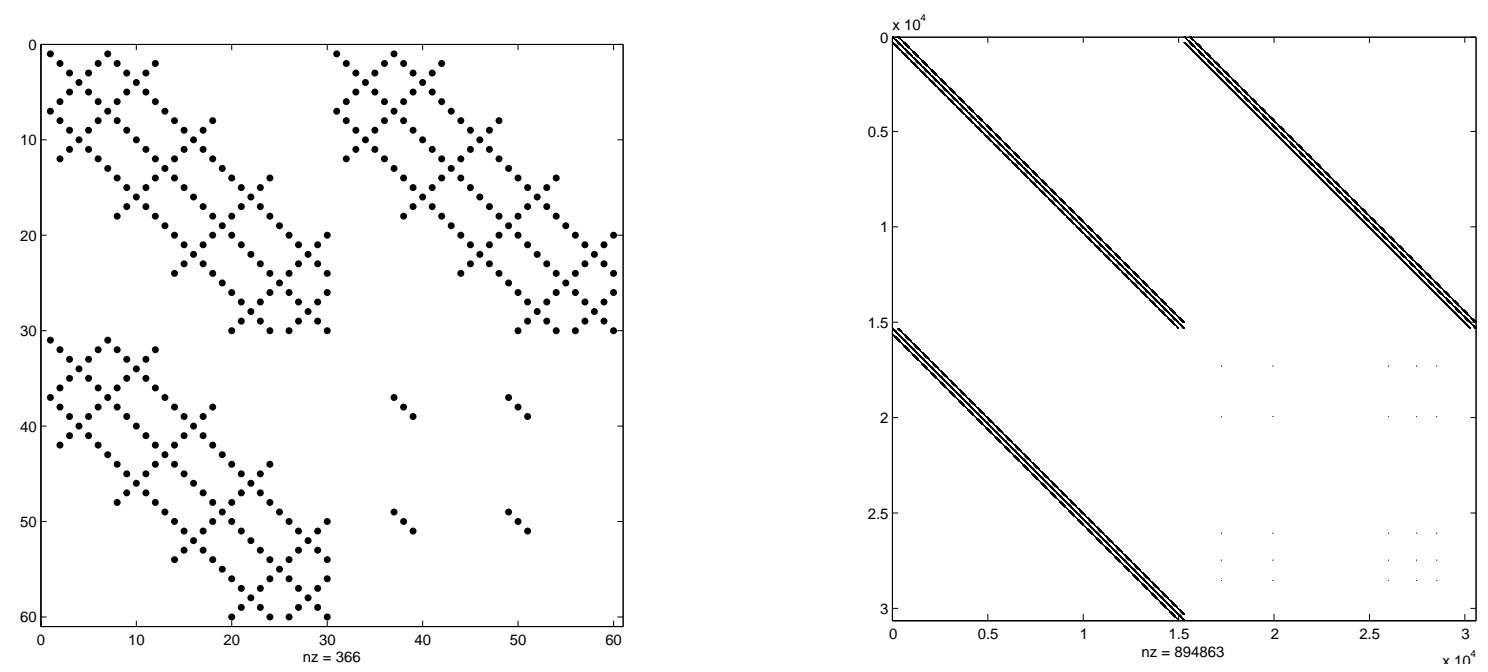

Figure 1: Position of non-zero terms of matrice $[A]$ with different size of FE model (left: low dofs, right: large dofs), without renumbering

Though renumbering techniques are evidently used in this work ${ }^{3}$, the decomposition operation is always the heavier one in the process.

\subsection{Robust expansion process: approach}

The expansion process depends evidently on the ability of the model to represent the identified structural dynamic behavior. This ability is based not only on the topology of the model (linear, viscoelastic, thermo-mechanical, etc) but also on the model input parameters which a subset is candidate for model calibration and denoted $p$. Furthermore, lack of knowledge is commonplace in complex FE model so that the choice of the calibration parameters must take the lack of knowledge in the system into account. Hence, robust expansion process requires not only to minimize the expansion error but also to enhance the ability of the model to be robust with regards to lack of knowledge in the system. To achieve this goal, info-gap theory is exploited [3]. In this work, we will study a system for which lack of knowledge is at parameter (local) levels. The uncertain parameters are denoted $u$ and are modeled by the info-gap relative error model (equation (8)), though a lot of other model are available (see [3] for more details):

$$
\mathcal{U}\left(\alpha, u^{0}\right)=\left\{u:\left|\frac{u-u^{0}}{u^{0}}\right| \leq \alpha\right\},
$$

where $\alpha \in \mathbb{R}_{+}$is the horizon of uncertainty which measures the distance - or the gap - between the estimate, $u^{0}$, and the whole possibilities in the $\operatorname{set} \mathcal{U}\left(\alpha, u^{0}\right)$. More precisely, the model of uncertainty $\mathcal{U}\left(\alpha, u^{0}\right)$ is an unbounded family of nested convex sets of realizable design. The unknown vector $u$ can represent pre-stress state, nonlinear constitutive relation, erroneous stiffness parameters, etc. Finally, the expansion process can be written as a function of $p$ and $u$, while $s$ denotes the expansion error:

$$
\mathcal{M}(p, u)=s, \quad u \in \mathcal{U}\left(\alpha, u^{0}\right)
$$

The expansion error is defined as the mean-relative error between the real mode shape $\left\{\phi_{\nu}^{*}\right\}$ and the expanded one $\left\{\hat{\phi_{\nu}}\right\}$ over the $N$ modes:

$$
s=\frac{1}{N} \sum_{\nu=1}^{N} \frac{\left\|\left\{\hat{\phi_{\nu}}\right\}-\left\{\phi_{\nu}^{*}\right\}\right\|}{\left\|\left\{\phi_{\nu}^{*}\right\}\right\|} .
$$

Let $s^{c}$ be the greatest level of error that the expansion process can tolerate:

$$
\mathcal{M}(p, u) \leq s^{c}
$$

The info-gap methodology is a decision theory: one of the main feature in this approach is the robustness function $\hat{\alpha}$. Broadly speaking, the robustness of decision $p$ is the greatest horizon of uncertainty that can be tolerated without exceeding

\footnotetext{
${ }^{3}$ In the case of large number of dofs, Metis renumbering method available in MD Nastran ${ }^{\circledR}$ permits to minimize the decomposition CPU-time
} 
the critical performance requirement $s^{c}$. In this article, the robustness function is expressed as a function of the design $p$ and the critical performance requirement $s^{c}$ and is written by:

$$
\hat{\alpha}=\hat{\alpha}\left(p, s^{c}\right)=\underset{\alpha \geq 0}{\arg \max }\left\{\max _{u \in \mathcal{U}\left(\alpha, u^{0}\right)}\{\mathcal{M}(p, u)\} \leq s^{c}\right\} .
$$

The robust design is simply the one which maximizes the robustness function:

$$
p^{R}=\underset{p}{\arg \max }\left\{\hat{\alpha}\left(p, s^{c}\right)\right\}
$$

\section{Numerical Applications}

The robust expansion process is illustrated on a simple academic cylinder. The question at stake in this study is: how to calibrate a non-validated model to both minimize expansion errors and still be robust with regards to lack of knowledge in the system. The FE model is depicted in Figure 2 while the experimental mode shape are simulated based on the real structure: the experimental mesh is composed of 300 dofs (cf. Figure 3).

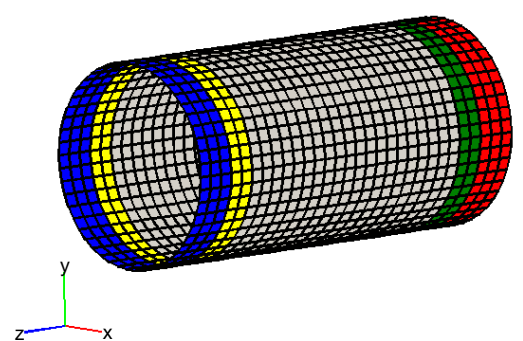

Figure 2: FE model

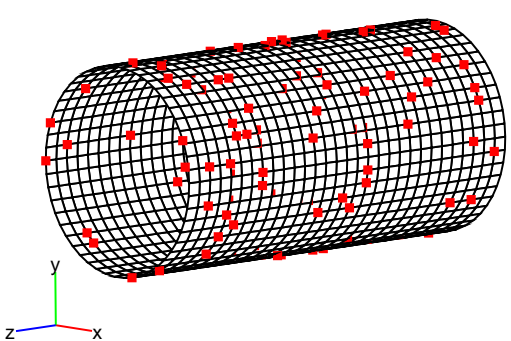

Figure 3: Random Sensor Locations

The cylinder is composed of five different zones: from zone 1 (corresponding to the direction $z>0$ ) to zone 5 (corresponding to the direction $z<0$ ). As expressed in the Table 1 , the difference between the real structure, which is used to simulate experimental eigensolutions, and the model one is located in zone 1,2,4 and 5. The zone 3, ie the medium zone, is supposed to be completely the same between the real structure and the model. The discrepancy between the numerical model and the real structure is also expressed in the Figure 4.

\begin{tabular}{|c|c|c|c|c|}
\hline Name: & Zone & Real & Model & Error \\
\hline Young Mod. E1 (Pa): & 1 & $3.10^{9}$ & $2,5.10^{9}$ & $-6 \%$ \\
\hline Young Mod. E2 $(\mathrm{Pa}):$ & 5 & $3.10^{9}$ & $2,2.10^{9}$ & $-13 \%$ \\
\hline Young Mod. E3 $(\mathrm{Pa}):$ & 2 & $2.10^{9}$ & $2,4.10^{9}$ & $+20 \%$ \\
\hline Young Mod. E4 $(\mathrm{Pa}):$ & 4 & $2,5.10^{9}$ & $2,7.10^{9}$ & $+8 \%$ \\
\hline
\end{tabular}

Table 1: Discrepancy Real/Model

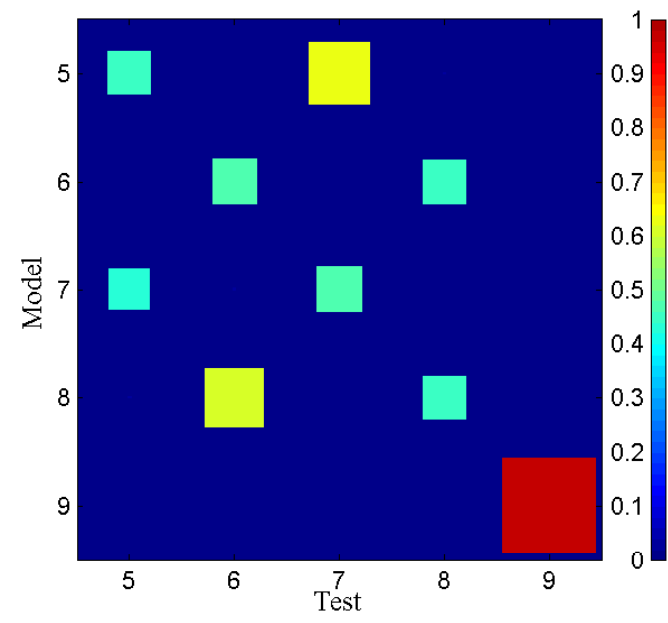

Figure 4: MAC between model and test

The parameters we supposed to be calibrated are E3 and E4 while the lack of knowledge is concentrated in parameters E1 and E2. Hence, definition of $u$ and $p$ (cf. section 2.2) are:

$$
u=\left[\begin{array}{ll}
E_{1} & E_{2}
\end{array}\right] \quad p=\left[\begin{array}{ll}
E_{3} & E_{4}
\end{array}\right] .
$$



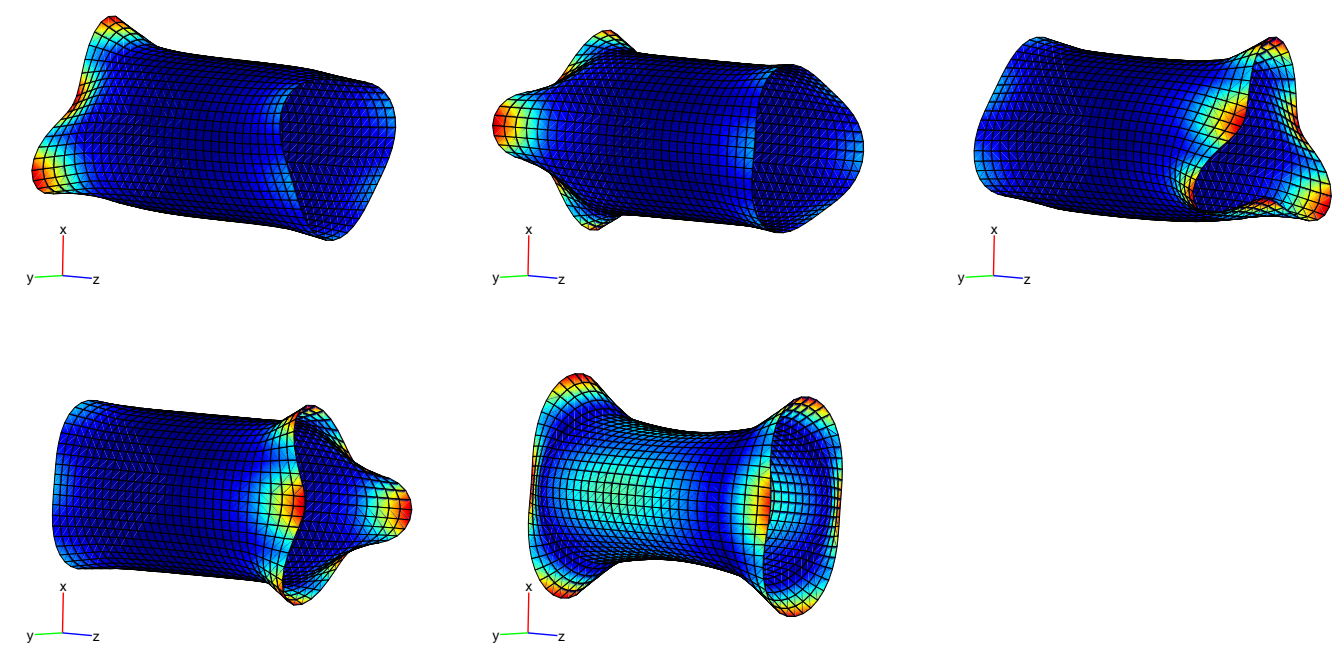

Figure 5: Real mode shapes

The mode shapes we seek to expand are composed of 4 local modes and 1 global mode as indicated in Figure 5.

When dealing with very simple model, which is the case for this study, using heuristic approach is sometimes faster and more reliable than optimization algorithm: for each step of horizon of uncertainty, the cost function is assessed at each born of the domain of uncertainty. In this particular case, two parameters are supposed to be uncertain: the domain of uncertainty is simply a square and thus the cost function has to be assessed four times (one time at each corner).

The nominal design is first analyzed in order to assess the robustness of the expansion to lack of knowledge in E1 and E2: at each horizon of uncertainty, the cost function is assessed four times, represented by the points and then the robustness curve is constructed by taking the maximum of the cost function (cf. Figure 6).

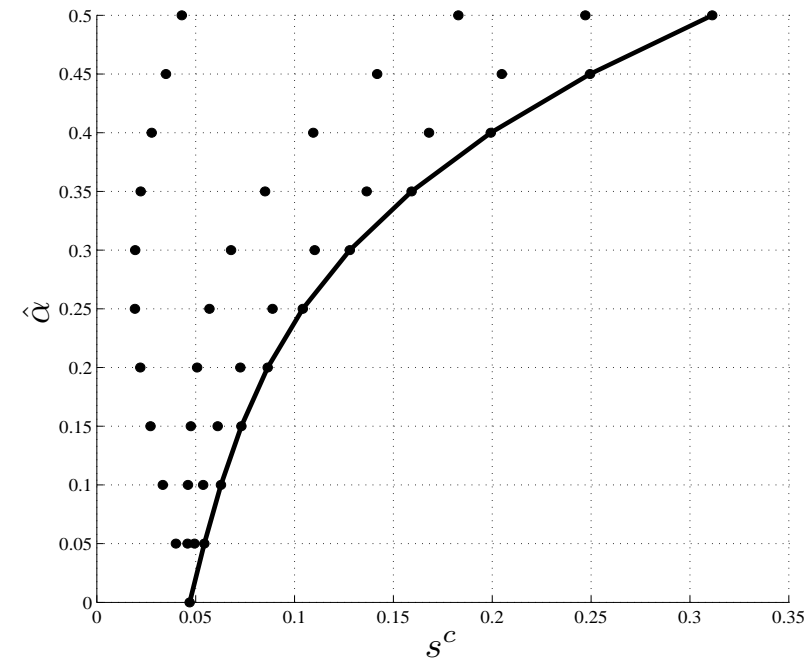

Figure 6: Robustness curve: nominal design

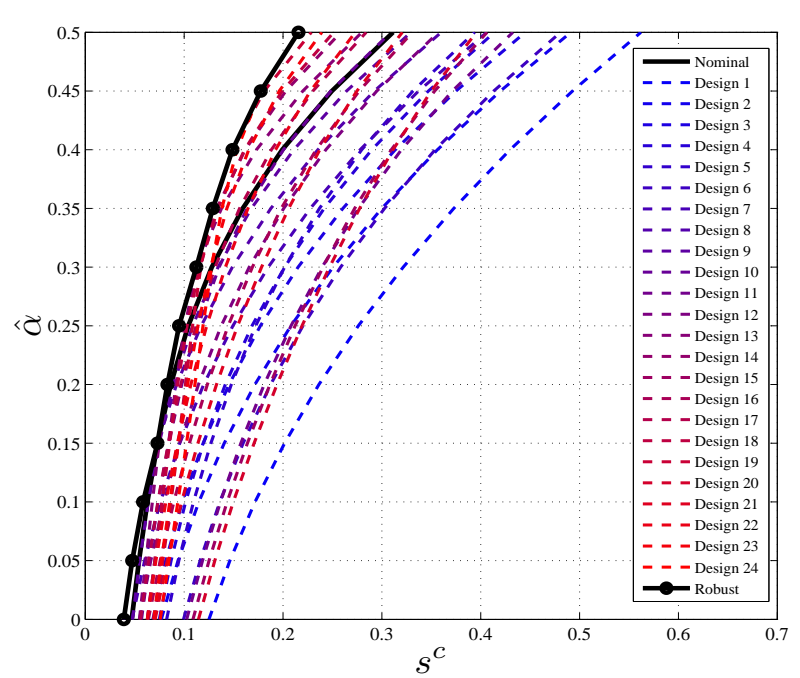

Figure 7: Robustness curves: investigation

Then, an investigation is performed based on a prior design space, depicted in Figure 8, in order to seek the most robust design, that is to say the one which maximizes the robustness function (equation (13)). In other words and concerning this particular case, the robust design minimizes the impact of lack of knowledge in the FE model (E1 and E2) on expansion errors. As above-mentioned, E3 and E4 are the model parameters we choose to calibrate: 24 designs are analyzed and the Figure 7 expresses their respective robustness. As expected, some designs are more robust than other ones. The robust 
design, ie the curve in black with filled black circle, is obtained after taking the minimum of $s^{c}$ at each $\hat{\alpha}$ step. This curve does not match with a particular fixed design but rather indicates the minimum error expected when uncertainty parameter is modeled with an info-gap model of uncertainty. Hence, the fixed robust design should be as close as possible to this curve in order to protect the model against uncertainty effects.

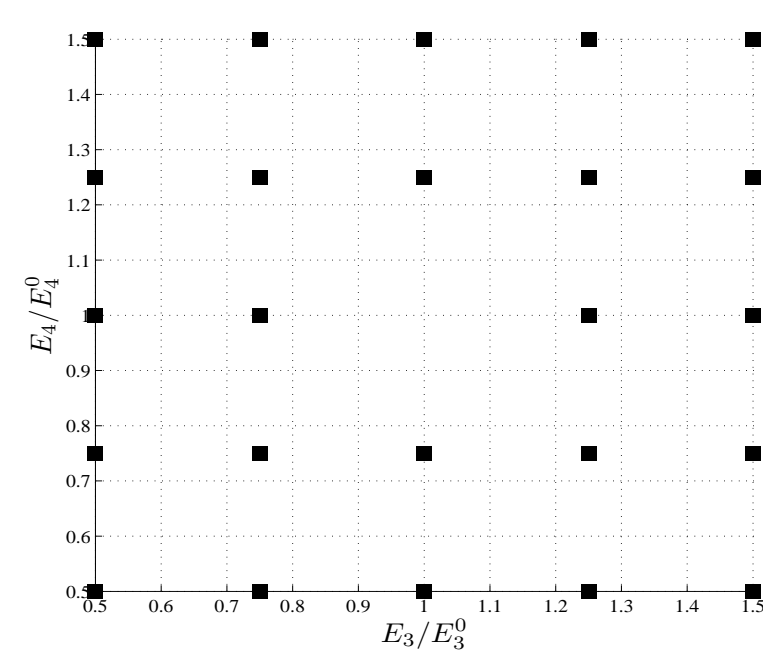

Figure 8: Design Space and the 24 tested design points

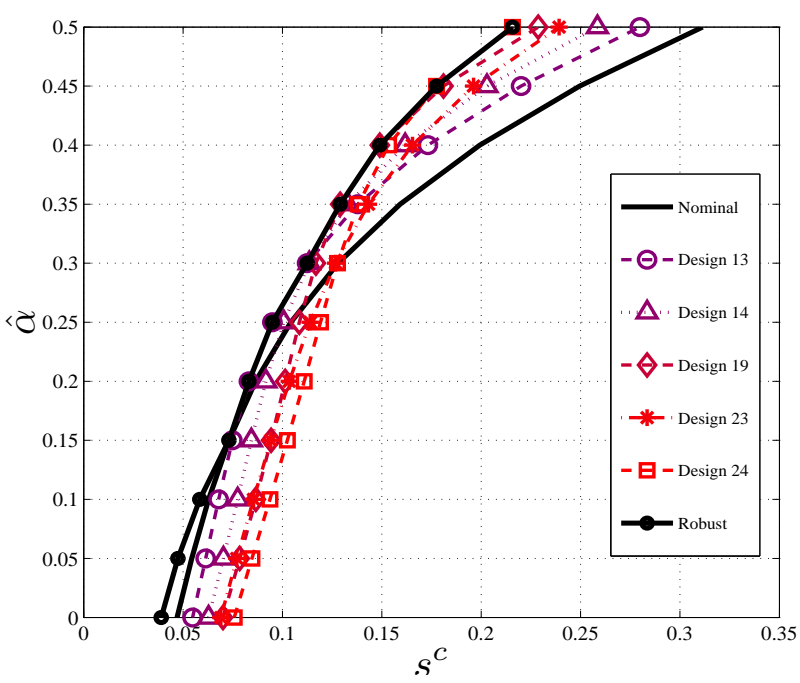

Figure 9: Robustness curves: selected designs

The Figure 9 proposes the robustness of the most robust fixed designs. For instance, if the experience feedback or the expert judgment pinpoints that the error in uncertainty parameters corresponds with an horizon of uncertainty closed to 0.5 , it would be preferable to use the design 24 in order to minimize the effect of lack of knowledge on the system model predictions.

\section{Conclusions}

The objectives of this work are both to assess the robustness of mode shape ECRE-based expansion on a nominal model in presence of large epistemic uncertainty and present a strategy, namely the robust model calibration, to minimize the impact of lack of knowledge in the model on expansion errors. After a state of art concerning both expansion methods and model calibration techniques, the formulation is presented. An academical application is then shown to illustrate the potential gains in terms of error: in the above example, the robust design, the design 24 , allows to around $10 \%$ less of expansion error at $\hat{\alpha}=0.5$. Though expansion errors are somewhat theoritical, output of the cost function can be, in a future work, maximum response levels in operation at the frequency band of interest. The ECRE-based expansion permits to obtain the extended mode shape after the resolution of a simple linear system. The main drawbacks of this technique remains the CPU-time. In a future work, model responses approximation techniques such as metamodeling will be used in order to reduce the time of computation.

\section{References}

[1] E. Balmès. Sensors, degrees of freedom and generalized mode expansion methods. In Proceedings of IMAC XVII, 1999.

[2] E. Balmès. Review and evaluation of shape expansion methods. In Proceedings of IMAC XVIII, 4062:555-561, 2000.

[3] Y. Ben-Haim. Information-Gap Theory: Decisions Under Severe Uncertainty. 2nd edition, Academic Press, London, 2006.

[4] Y. Ben-Haim and F. Hemez. Robustness, fidelity and prediction-looseness of models. Proceedings of the Royal Society A, 468:227 - 244, 2011. 
[5] M. Corus and E. Balmès. Improvement of a structural modification method using data expansion and model reduction techniques. In Proceedings of IMAC XXI, 2003.

[6] A. Deraemaecker. Sur la maîtrise des modèles en dynamique des structures à partir de résultats d'essais. PhD thesis, École Normale Supérieure de Cachan, 2001.

[7] A. Deraemaeker, P. Ladevèze, and P. Leconte. Reduced bases for model updating in structural dynamics based on constitutive relation error. Computer methods in applied mechanics and engineering, 191:2427-2444, 2002.

[8] P. Feissel and O. Allix. Modified constitutive relation error identification strategy for transient dynamics with corrupted data: The elastic case. Computer methods in applied mechanics and enginnering, 196:1968-1983, 2007.

[9] R. Guyan. Reduction of stiffness and mass matrices. American Institute of Aeronautics and Astronautics Journal, 3:380, 1964.

[10] F. Hemez and Y. Ben-Haim. Info-gap robustness for the correlation of tests and simulations of a non-linear transient. Mechanical Systems and Signal Processing, 18:1443-1467, 2004.

[11] R. Kidder. Reduction of structural frequency equations. AIAA Journal, 11 (6), 1973.

[12] P. Ladevèze. Comparaison des modèles des milieux continus. PhD thesis, Université Pierre-et-Marie-Curie, 1975.

[13] S. Michot. Contributions à la validation de modèles en dynamique des structures. PhD thesis, Université de FrancheComté, 2003.

[14] J. Mottershead and M. Friswell. Model updating in structural dynamics: A survey. Journal of Sound and Vibration, 167(2):347-375, 1993.

[15] J. O'Callahan. A procedure for an improved reduced system (irs) model. Proceedings of IMAC VII, pages 17-21, 1989.

[16] J. O'Callahan, P. Avitabile, and R. Riemer. System equivalent reduction expansion process (serep). In Proceedings of IMAC VII, pages 29-37, 1989.

[17] R. Pascual. Model Based Structural Damage Assessment Using Vibration Measurements. PhD thesis, Université de Liège, 1999.

[18] R. Pascual, J. Golinval, and M. Razeto. On the reliability of error localization indicators. In Proceedings of the ISMA23, 1998.

[19] D. Pereiro, S. Cogan, E. Sadoulet-Reboul, and F. Martinez. Robust model calibration with load uncertainties. In Proceedings of the IMAC XXXI, 2013.

[20] M. Reynier. Sur le contrôle de modélisations par éléments finis: recalage à partir d'essais dynamique. PhD thesis, Université de Paris VI, 1990. 